\title{
LA CARIDAD COMO CRITERIO HERMENÉUTICO. UNA APROXIMACIÓN A LA COMPRENSIÓN DE LA UNIDAD DEL $D E$ DOCTRINA CHRISTIANA DE SAN AGUSTÍN
}

\author{
Biviana Unger Parra* \\ doi:10.11144/Javeriana.uph32-64.cchc
}

\section{RESUMEN}

El De doctrina christiana es una de las obras más importantes de San Agustín, no solo por la reflexión y el trabajo hermenéutico que propone, sino por su particular carácter, pues su composición da cuenta del desarrollo vital e intelectual del autor a lo largo de dos decenios. Un gran sector de la crítica afirma que esta obra debe entenderse como un conjunto formado por dos partes irreconciliables entre sí, aquella escrita en el 396, de carácter hermenéutico y, aquella compuesta en el 426 dedicada a la exposición de la retórica y la doctrina de la gracia. Si bien no debe desconocerse la diferencia que existe entre las dos partes de la obra, es necesario buscar un punto de confluencia del cual no solo depende la unidad de la obra, sino también la de la entera cultura cristiana.

Palabras clave: De doctrina christiana; Agustín; caritas; hermenéutica; retórica

* Pontificia Universidad Javeriana, Bogotá, Colombia.

Correo electrónico: bivianaunger@gmail.com

Para citar este artículo: Unger ParRa, B. (2015). La caridad como criterio hermenéutico. Una aproximación a la comprensión de la unidad del De doctrina christiana de San Agustín. Universitas Philosophica, 32(64), pp. 329-341, ISSN 0120-5323, ISSN en línea: 2346-2426, doi: 10.11144/ Javeriana.uph32-64.cchc 


\title{
CHARITY AS A HERMENEUTIC CRITERION. AN APPROXIMATION TO THE COMPREHENSION OF THE UNITY OF ST. AUGUSTINE'S DE DOCTRINA CHRISTIANA
}

\author{
Biviana Unger UngeR
}

\begin{abstract}
The De Doctrina Christiana is one of St. Augustine's most important works, not only because of the reflection and hermeneutic exposed, but because of its special nature, since their composition realizes the vital and intellectual development of the author over two decades. A large section of the specialized reviews say that this work should be understood as a set of two irreconcilable parts, that one written in 396, with a hermeneutical character, the other finished by 426 devoted to rhetoric and the doctrine of grace. While it should not be ignored the difference between these two parts of the work, it is necessary to find a common ground that not only ensures the unity of the work, but also of the whole Christian culture. Key works: De doctrina christiana; St. Augustine; caritas; hermeneutics; rhetoric
\end{abstract}


El De DoCTRINA CHRISTLANA EMPezó a gestarse en el año 396, aunque no se completaría sino luego de 30 años. Se trata de una de las primeras grandes obras agustinianas que, gracias al carácter particular de su composición, nos permite asistir al proceso de culminación de un pensamiento que se desarrolla durante dos decenios. Un amplio sector de la crítica ha considerado la interrupción de la composición en términos de una fractura insalvable entre las dos partes de la obra, queriendo así sostener la renombrada tesis acerca de las diferentes etapas del pensamiento agustiniano, conocidas como conversiones. En contra de dicho sector, mi intención es la de demostrar la unidad de la obra a partir de la consideración de la noción de caridad, fundamental no solo para la comprensión del $D e$ doctrina christiana y la defensa de su unidad, sino de toda la obra agustiniana, constituyéndose así en criterio hermenéutico de la misma.

En las primeras obras de Agustín, consideradas como la clave de su pensamiento filosófico, aparece de manera constante la cuestión del lugar que deben ocupar las disciplinas en el conjunto formativo del cristiano. Las artes liberales, punto de referencia de la sabiduría clásica, ocuparán un lugar fundamental en las primeras obras, sobre todo, en los Diálogos de Casiciaco y en el proyecto intelectual de los primeros años de producción agustiniana. No sorprende entonces que, en esta misma época, Agustín se haya dado a la tarea de componer un libro llamado Sobre las disciplinas, dedicado al estudio de la gramática, la retórica, la dialéctica, la aritmética, la geometría, la música y la filosofía. Dicho proyecto no tuvo culminación y solo se terminó el texto acerca de la gramática y se avanzó en aquel dedicado a la música, del cual únicamente se escribieron seis libros dedicados al ritmo y no aquellos sobre la melodía. Aunque se desconocen las razones por las cuales no se llevó a cabo este proyecto, es imposible desconocer la importancia de las artes liberales en el pensamiento de Agustín, si bien, con el paso del tiempo, se irá adoptando una posición según la cual el cristianismo se rige por sus propias reglas y estas no siempre coinciden con las del humanismo clásico.

A propósito de esto, una especial mención merece el diálogo De ordine, en el cual se ofrece un interesante panorama de las artes liberales y la manera en la que estas se ponen al servicio de la comprensión de la realidad. De este modo, la gramática, la dialéctica y la retórica aparecen como momentos fundamentales de un camino de ascenso que ha de recorrerse si se busca el verdadero conocimiento. En De ordine se dan las bases para la construcción de una paideia cristiana que 
encuentra concreción, algunos años después y bajo unas circunstancias particulares, en el De doctrina christiana, pues se trata de la primera obra compuesta en calidad de obispo de Hipona. Este dato no debe tomarse a la ligera, ya que existe una íntima relación entre las razones de la composición del De doctrina christiana y la situación por la cual estaba atravesando la iglesia norteafricana. Así pues, podemos afirmar que se trata del primer fruto de la experiencia vivida y de los objetivos trazados en sus años como presbítero y de su nueva condición de obispo, tales como el combate de las herejías y el acercamiento de todos los cristianos a las Sagradas Escrituras. Agustín mismo había sentido la dificultad de la interpretación de las Escrituras, pues su estilo sencillo y su lenguaje rudo se alejaban de la elegancia y majestuosidad de los textos clásicos a los que él estaba acostumbrado. A propósito de lo anterior se afirmará en las Confesiones: "mi hinchazón recusaba su estilo y mi mente no penetraba su interior. Con todo ellas eran tales que habían de crecer con los pequeños; mas yo me desdeñaba de ser pequeño, e hinchado de soberbia me creía grande" (Agustín, 1946, III, 5, 9). A causa de la soberbia, el joven Agustín no entendía que el estilo llano de la Escritura, asequible a todos, corresponde a la naturaleza sencilla y limitada de la humanidad. Sin embargo, la interpretación de la misma es un proceso que no todos pueden llevar a cabo, puesto que se requiere de años de estudio y preparación. Por esta razón, para llevar a cabo un ajuste de cuentas con la tradición retórica ${ }^{1}$ era necesario poner por escrito las reglas hermenéuticas y exegéticas necesarias para enfrentarlas, así como reconocer el valor retórico de las mismas. Por otra parte, resultaba apremiante establecer, frente a las herejías, las líneas del cristianismo en cuanto a su relación con la cultura pagana. En este punto, surgió la cuestión de qué herramientas debían usarse en el proyecto de la constitución de una cultura cristiana. El problema crucial consistía en establecer si la Iglesia debía adoptar o no la cultura latina que Roma había heredado de Grecia, cuestión respecto de la cual no existía una postura uniforme. En los primeros siglos, algunos de los nuevos cristianos que habían sido educados bajo los cánones de la cultura clásica, en el momento de la conversión, rechazaron su formación y tomaron una actitud hostil

1 En el libro IV del De doctrina christiana se afirma que los autores cristianos habían hablado con la elocuencia conveniente al tema por ellos tratado, mostrando un perfecto equilibrio entre estilo y contenido. Se sostiene también que todas las técnicas retóricas conocidas se encuentran ya en las Sagradas Escrituras, aunque su uso es moderado y armónico en todas las ocasiones. 
frente al saber pagano. El recelo de la Iglesia frente a los escritores, poetas y filósofos clásicos se puede ver con claridad en la prohibición del IV Concilio de Cartago (398), según la cual los obispos no podían leer los libros de los gentiles, a menos de que fuera necesario. ¿Qué posición asume el hiponense frente a esta cuestión? La estructura misma del De doctrina christiana nos da una primera luz para responder a este interrogante, pues sigue la división retórica clásica que distingue entre modus inveniendi y modus proferendi, esto es, el modo de encontrar lo que se debe entender y el modo de explicar lo que se ha entendido. Así, los tres primeros libros, que componen la primera parte, se ocupan del reconocimiento de las reglas necesarias para la elaboración de una hermenéutica bíblica, mientras el último libro aborda la cuestión de la transmisión de las reglas reconocidas.

El primer paso consiste en una distinción retórica, presente ya en Cicerón, entre cosas y signos, que afirma que toda instrucción se reduce a la enseñanza de cosas y de signos (res y verba). Tanto para Agustín como para Cicerón, el hombre está inmerso en un mundo simbólico en el que la relación signo-cosa se constituye como un esquema dentro del cual se da tanto el conocimiento como la comunicación. De hecho, la doctrina existe gracias al signo y debe entenderse como la determinación cognoscitiva de la res. Una vez hecha la anterior distinción, se pasa a determinar en qué consiste el ámbito de la res, pues a partir de aquí se da inicio a la comprensión que constituye la base de la hermenéutica cristiana. En primer lugar, se sostiene que hay tres tipos de cosas: aquellas de las que se debe gozar; las que deben ser uasdas y, por último, las que pueden ser objeto de gozo y uso a la vez. Nos encontramos aquí frente al segundo par dialéctico presente en el De doctrina christiana: uti-frui. La definición que se ofrece de ambos conceptos es la siguiente: "gozar es adherirse a una cosa por el amor de ella misma. Usar es emplear lo que está en uso para conseguir lo que se ama, si es que debe ser amado" (Agustín, 1991, I, 4, 4). Esta distinción cumple un papel fundamental en el estudio que hace Agustín acerca de la diferencia entre el amor hacia Dios y el amor hacia el prójimo, donde se pregunta si el hombre debe amar al prójimo por él mismo o en virtud de otra cosa. La respuesta que se ofrece es clara: nadie debe amar al prójimo por él mismo y tampoco "nadie debe amarse a sí mismo, sino por aquel de quien debe gozar” (Agustín, 1991, I, 22, 21). Las cosas que han de amarse están, entonces, organizadas según cuatro géneros. El primero concierne a aquello que está sobre el hombre; el segundo tiene que ver con el hombre mismo; el tercero, con 
su prójimo, y el cuarto, con lo que está debajo del hombre. En este punto, se llama la atención sobre el hecho de que no es necesario que exista precepto alguno con respecto al amor que el hombre debe tener de sí mismo y de las cosas inferiores a él, pues aun cuando el ser humano se aparta de la verdad, mantiene el amor por su alma y por su cuerpo, así como por todo aquello que le pertenece. Entonces, debe tomarse en consideración el precepto dado para que el hombre ame lo que está sobre él y junto a él, precepto condensado en las palabras de Mateo: "amarás a tu Dios y Señor con todo tu corazón, con toda tu alma y con toda tu mente; y a tu prójimo como a ti mismo. En estos dos preceptos se incluye toda le ley y los profetas" (Mat. 22:37). ¿Cómo se conjuga el tema del amor a Dios y al prójimo con el problema que nos concierne? ¿Por qué afirmamos que este amor se configura como criterio hermenéutico? Según las palabras de Agustín, "El compendio de todo lo expuesto desde que comenzamos a tratar de los objetos o cosas, es entender que la esencia y el fin de toda la divina Escritura es el amor de la Cosa que hemos de gozar y de la cosa que con nosotros puede gozar de Ella, pues para que cada uno se ame no hubo necesidad de precepto" (Agustín, 1991, I, 35, 39).

$\mathrm{Al}$ afirmar que el fin de la Escritura es la caridad, esta se constituye como criterio interpretativo, asumiendo así un doble movimiento que parte de la ética, pero que se realiza en la hermenéutica. Podemos afirmar con Lettieri que, desde este punto de vista, el fin de la doctrina cristiana y de la actividad exegética es el intellectus de la caritas, esto es, la capacidad intelectual y moral de subordinar el uti de los signos sensibles al frui de la Res inmutable. Esta es la clave de la interpretación, la cual se completa en el docere, razón por la cual la retórica es el segundo momento de la hermenéutica, tal y como se había ya explicado con respecto a la estructura misma del De doctrina christiana. Así pues, el intellectus de la caritas debe entenderse como un movimiento, a la vez, ético y hermenéutico, pues existe una íntima comunión entre llevar una vida buena, comprender y amar. A propósito de esto, Pollmann afirma que la caritas - ética forma el horizonte normativo para la comprensión de la Escritura y da los preceptos para la vida del hombre, constituyéndose, al mismo tiempo, como meta y condición. La anterior relación había sido establecida con claridad en el De moribus ecclesiae catholicae et de moribus Manicheorum donde se afirma que así como la virtud es el camino que conduce a la verdadera felicidad, esta última no es otra cosa que un perfecto amor a Dios (Agustín, 2011, I,6,9). Así, las cuatro virtudes cardinales deben en- 
tenderse como funciones diferentes del amor. De este modo, la templanza es amor que conoce su propia integridad; la justicia es amor que desea compartir aquello que es amado; por último, la prudencia se entiende como el amor que con sagacidad es capaz de elegir los mejores medios para llegar a Dios y no alejarse de Él. El fin de las cuatro virtudes no es otro que la unión con Dios, a la cual se llega a través de la caridad que subyace a cualquier modo de virtuosismo. En este punto, Agustín une caridad, virtud y sabiduría retomando las palabras del Apóstol, según las cuales el Hijo es Virtud y Sabiduría de Dios, y las del libro de la Sabiduría, donde se afirma que la sabiduría nos enseña la templanza, la justicia y la fortaleza, en cuya comparación nada hay más útil para la vida de los hombres (Sab. 8:1). La caridad está en el centro de la espiritualidad agustiniana en cuanto es la medida de la justicia en el corazón de los hombres, de manera que a una caridad incipiente corresponde una justicia incipiente, mientras una caridad en aumento determina el aumento de la justicia y, en este orden de ideas, una caridad elevada corresponde a una justicia elevada.

Una vez más, vemos cómo la caridad es la condición fundamental para llegar al conocimiento de Dios, ya que si la sabiduría y la verdad no se aman con todas las fuerzas del espíritu no se puede, en modo alguno, llegar a conocerlas; pero, si se busca como se debe, no se esconde a sus amantes. En consonancia con la anterior afirmación debe entenderse la declaración que aparece dos veces en las Confesiones, donde Agustín afirma: "por amor de tu amor hago esto" (Agustín, 1946, II, 1,1).

Para entender mejor el valor ético-hermenéutico de la noción de caridad hay que tener en cuenta también su carácter escatológico. La caridad tiene un valor fundamental en la terna que conforma junto con la esperanza y la fe, debido a que estas últimas no trascienden la vida terrena, mientras que la caridad es tanto inmanente como trascendente. La caridad agustiniana se aleja de la noción griega de eudaimonía, en cuanto que en el platonismo la definitiva realización de la felicidad solo es posible para el alma después de la muerte corporal, mientras que para Agustín es todo el hombre, fuera de la historia, el que alcanza la felicidad eterna.

Esta primera aproximación a la relación existente entre hermenéutica y ética, cuyo indiscutible punto de unión es la caridad, nos permite acceder a una comprensión del carácter del De doctrina christiana que va más allá de la consideración de esta como un manual técnico en el que se exponen las reglas de la interpretación. Así pues, diremos en contra de aquellos que ven una insalvable 
fractura entre las dos partes de la obra ${ }^{2}$, que es justamente la caridad la categoría que funciona como eje entre los libros dedicados a la exposición y aquellos dedicados a la difusión y comunicación de los preceptos interpretativos. Retomando una idea clásica, y uniendo la figura del exegeta y el orador, Agustín considera que el buen intérprete y el buen rétor debe ser al mismo tiempo un hombre recto, que refleje su ethos en sus palabras. De manera que aquel que actúa según la fe, la esperanza y la caridad, los tres principios de la vida del cristiano, se encuentra en capacidad de instruir a los demás, pues posee la perfección mayor a la cual puede aspirar el hombre en la vida terrena. Por este motivo, en la introducción al cuarto libro del De doctrina christiana se ofrece una amplia justificación de la necesidad de la retórica como instrumento de fe. Para Agustín, siguiendo un precepto ya presente en Cicerón, la belleza y la eficacia de la expresión estaban íntimamente ligadas a sus contenidos, y es por esta razón que la formación del rétor es un proceso que va más allá de la simple práctica y que involucra distintas habilidades (Cicerón, 2002, II, 44). Según el método clásico, la preparación de un experto en retórica se da en tres momentos: en primer lugar, se encuentra el aprendizaje de las reglas (praecepta); en segundo lugar, la imitación de los modelos (imitatio) y, por último, la composición libre acerca de un tema preestablecido (declamatio). En consonancia con la importancia otorgada al contenido, el momento más importante de la preparación del rétor es el de la imitación, puesto que a través de la práctica se adquiere la elocuencia, fundamental en el efecto esperado sobre el auditorio. Aquí es decisiva la experiencia pastoral del obispado y la necesidad de establecer con claridad la manera en la que debía usarse la retórica. Siguiendo, una vez más, a Cicerón, se afirma que el hombre locuaz debe hablar para enseñar (ut doceat), deleitar (ut delectet) y persuadir (ut flectat), lo cual es, para el orador: deber, placer y victoria. (Cicerón, 2002, I, 87)

Desde esta misma perspectiva debe entenderse la doctrina semántica agustiniana, desarrollada en la primera parte de la obra en cuestión, la cual, si bien tiene un fundamento estoico, está signada por un trasfondo metafísico desde el que es

2 En L'altro Agostino. Ermeneutica e retorica della grazia dalla crisi alla metamorfosi del De doctrina christiana, Gaetano Lettieri afirma que debemos considerar que en el interior De doctrina christiana encontramos dos obras diferentes pertenecientes a periodos distintos del pensamiento agustiniano, en el cual se pueden identificar con claridad dos momentos distintos determinados por la aparición de la doctrina de la gracia. Véase al respecto: Lettieri, 2002 pp. 85ss. 
posible concebir la constitución misma de la realidad, ontológicamente bipartita. Así, por una parte se encuentran las cosas y, por otra, sus signos, los cuales derivan su ser del signo fundamental, a saber: Cristo, de la misma manera que las cosas derivan su ser de la potencia creadora de Dios. La mediación del signo se hace necesaria por la constitución misma de la naturaleza humana y, en este sentido, debe entenderse como "el precio provisional que la humanidad pecadora debe pagar en su camino hacia la verdad" (Alici, 2002, p. 32). Sin embargo, esto no significa que en la vida terrena sea imposible trascender la esfera de lo meramente sígnico. De hecho, el paso de un ámbito a otro, del signum a la res, se da gracias a la caridad, pues "estando arraigados y cimentados en la caridad, podemos comprender con todos los santos, cuál sea la anchura, la longitud, la altura y la profundidad, esto es, la Cruz del Señor" (Agustín, 1991, II, 41, 62). La caridad se presenta aquí con todo su valor, tanto hermenéutico como existencial ${ }^{3}$. La venida de Cristo le permite al hombre no solo librarse de la esclavitud de la ley, sino también de los signos, lo cual posibilita una nueva hermenéutica que trascienda el ámbito de los signa y se sitúa en el de la res. Pero, ¿̇cómo se da este paso? Gracias a un llamado de amor. Este llamado se caracteriza por ser siempre eficaz, dado que Dios, rétor supremo, a diferencia del rétor humano, distingue a los elegidos mediante un tipo determinado de llamado, el apto para cada caso. Esta consideración del llamado de Dios como retórica nos permite establecer una relación entre revelación y estilos retóricos, ya que aquella se da de tres maneras: primero, según el estilo sencillo y explicativo, esto es, a manera de doctrina; segundo, de forma moderada, por medio de la cual se puede llamar a muchos pero no persuadirlos y, tercero, de manera elevada, donde el Espíritu persuade a través del Verbo infalible.

De este modo, tanto la hermenéutica como la retórica son instrumentos de los cuales la gracia ${ }^{4}$ se sirve para actuar, razón por la cual ambas son producto de la acción liberadora de Cristo. Así pues, debemos concluir que en la cruz, signo por excelencia de la vida del cristiano, encontramos el punto de unión entre her-

3 Alici y Lettieri llaman la atención sobre la gran influencia que esta característica de la hermenéutica agustiniana ha tenido en la filosofía contemporánea, de modo especial en Gadamer.

4 La relación entre hermenéutica, retórica y gracia merece una profundización que excede el propósito del presente escrito. Un texto de referencia obligado es el ya citado L'altro Agostino de Gaetano Lettieri. 
menéutica y retórica y, por lo tanto, entre las dos partes del De doctrina christiana. Por una parte, Cristo como Verbo encarnado constituye el fundamento sígnico del lenguaje humano y, por otra, es el maestro interior que a través del Verbo siempre eficaz llama y persuade al hombre. Este doble movimiento se halla ya evidenciado en el título mismo de la obra, pues es claro que la doctrina hace referencia al conjunto de los signos por medio del cual está expresada la Escritura, que debe ser estudiada y entendida (modus inveniendi) por el exegeta; mientras que al decir que esta doctrina es cristiana se hace alusión a la exigencia del cristiano de transmitir la revelación de dicho llamado (modusproferendi). Con esto, se da una clave de lectura al interrogante que suscita la distancia entre los dos momentos de composición de la obra y, con ello, un paso a favor de la unidad de la misma. Esta última es fundamental en cuanto nos ayuda a comprender la dinámica interna del pensamiento de Agustín y de toda su obra, de manera que el De doctrina christiana se presenta como compendio de sus principales posturas filosóficoteológicas. Así, en los tres primeros libros tenemos la concreción de un pensamiento que recoge los principales motivos de la cultura clásica, mientras que con el cuarto asistimos a la apropiación cristiana de dicho pensamiento, lo que nos muestra que esta obra no solo trata el tema de la hermenéutica, sino que ella misma es producto de un ejercicio hermenéutico: el de Agustín como autor. Teniendo en cuenta lo anterior, sorprende la posición de aquellos autores que afirman que existe una incompatibilidad teológica y doctrinaria entre las dos partes de la obra y que la decisión de terminarla no obedeció a una razón particular, sino a un simple interés por terminar un texto inconcluso pues, con base en lo que hasta aquí hemos demostrado, la obra tiene una unidad clara que depende de la intención inicial de Agustín y del ejercicio hermenéutico que él mismo lleva a cabo en relación con su producción intelectual. Esto no significa que deba desconocerse que en los años de la interrupción del De doctrina christiana se llevó a cabo el perfeccionamiento de la doctrina de la gracia y que, sin duda alguna, esta ofrecerá un nuevo horizonte comprensivo desde el cual se abordarán las más hondas preocupaciones. ¿Cuál es, entonces, la relación que existe gracia y caridad? ¿Debemos pensar que la configuración de la doctrina de la gracia se contrapone a la consideración de la caridad como criterio hermenéutico? Para resolver estos interrogantes es importante tener presente que la gracia es la actuación de Dios al interior de los hombres, la cual se traduce en un llamado de amor que 
mueve a los hombres a amar a Dios como el bien supremo de toda la creación. Del amor ordenado a Dios se sigue el amor hacia el prójimo, hacia sí mismo y hacia todos los bienes inferiores, lo que nos muestra que la gracia conjuga los cuatro tipos de amor en el primer libro del De doctrina christiana. Así pues, también se puede concluir que la doctrina de la gracia incluye y perfecciona la doctrina de la caridad, y que uno de los lugares en donde se lleva a cabo dicho perfeccionamiento es la parte conclusiva del De doctrina christiana. Es por esta razón que el libro cuarto de esta obra es una síntesis de los elementos retórico-hermenéuticos trabajados en los tres primeros libros y de la doctrina de la gracia desarrollada en algunas de las obras compuestas en los 30 años que espacian las dos partes del texto, entre las que merece especial mención el De diversis Quastionibus ad Simplicianum, compuesta al parecer, en la primera mitad del año 397. Esta última, si bien trata los temas del libre albedrío, la justificación y la gracia, nace de un interés hermenéutico, como lo podemos constatar en el prólogo:

Has hecho llegar hasta mí, ¡oh padre mío Simpliciano!, el envío agradabilísimo y delicadísimo de tu cuestionario; y si no procurara satisfacerte, no sólo sería desobediente, sino también ingrato. Ya he discutido y publicado algo sobre las dificultades que me has propuesto de algunos pasajes del apóstol San Pablo. Mas, poco satisfecho de mi primera investigación y comentario, y a fin de evitar descuidos y omisiones, he vuelto a someter a un examen más diligente y serio las mismas palabras y razonamientos del Apóstol. Si su interpretación fuera obvia y fácil, no las creerías tú dignas de nueva discusión. (Agustín, 1952, I)

Nótese que la terminología hermenéutica en la cual se expone el propósito de la obra es muy cercana a la utilizada en el De doctrin a christiana y, teniendo en cuenta que lo que se hace en el De diversis Quastionibus ad Simplicianum es interpretar los pasajes oscuros de las cartas de San Pablo, poniendo especial atención en las palabras y el tenor de sus sentencias, podemos afirmar que la intención de ambas obras es la misma. De este modo, la divergencia no radica en el fin sino en los medios, pues, mientras el De doctrina christiana puede considerarse una síntesis de la paideia latina con elementos cristianos, el texto dirigido a Simpliciano une los mismos elementos clásicos con un componente novedoso y específico: la doctrina de la gracia. Por otra parte, de la identificación del fin de las dos obras también puede deducirse que el De diversis Questionibus ad Simplicianum 
es la aplicación específica de la hermenéutica desarrollada en el 396. De esta última se retoma, en especial, la distinción de los signos entre metafóricos y propios, así como la utilización de la interpretación alegórica en los casos en los que sea necesaria para la interpretación de un pasaje oscuro. Aunque el marco hermenéutico fundamental parece haber cambiado, pues ya no se habla de caridad sino de gracia, ya vimos cómo una doctrina se recoge dentro de la otra, completándose y adoptando un sentido nuevo.

De este modo, podemos afirmar que el De doctrina christiana sintetiza los dos grandes momentos de la vida de Agustín: su formación clásica y su condición cristiana, los cuales, a su vez, se constituyen en fundamento de la entera modernidad.

\section{Referencias}

Agustín, santo obispo de Hipona. (1946). Confesiones. Madrid: Biblioteca de Autores Cristianos.

Agustín, santo obispo de Hipona. (1952). Sobre diversas cuestiones a Simpliciano. Madrid: Biblioteca de Autores Cristianos.

Agustín, santo obispo de Hipona. (1991). Sobre la doctrina cristiana. Escritos bíblicos, vol XV. Madrid: Biblioteca de Autores Cristianos.

Agustín, santo obispo de Hipona. (1994). El orden. Escritos Filosóficos, vol. I. Madrid: Biblioteca de Autores Cristianos.

Agustín, santo obispo de Hipona. (2011). Las costumbres de la Iglesia y la de los maniqueos. Escritos apologéticos, vol. IV. Madrid: Biblioteca de Autores Cristianos.

Alici, L. (2002). Veritá e linguaggio. Agostino nella filosofia del Novecento. Roma: Città Nuova.

Bright, P. (1995). Biblical Ambiguity in African Exegesis. De doctrina Christiana. A Classic of Western Culture. Indiana: University of Notre Dame Press.

Cicerón, M.T. (2002) Sobre el orador. Madrid: Gredos.

Lettieri, G. (2001). L'altro Agostino. Ermeneutica e retorica della grazia dalla crisi alla metamorfosi del De doctrina christiana. Brescia: Morcelliana. 
Mayer, C. (1985). Prinzipien der Hermeneutik Augustins und daraus sich ergebende Probleme. Forum Katholische Theologie, 1, pp.197-211.

Pollmann, K. (1996). Doctrina Christiana. Untersuchungen zu den Anfägen der christlichen Hermeneutik unter besonderer Berücksichtigung von Augustinus De doctrina Christiana. Friburgo: Universitätsverlag Freiburg. 